\title{
Nonlinear Optical Loop Mirror Based on Standard Communication Fiber
}

\author{
Ding Wang, Ekaterina A. Golovchenko, Alexei N. Pilipetskii, Curtis R. Menyuk, Senior Member, IEEE, \\ and Mark F. Arend
}

\begin{abstract}
We numerically analyze the effectiveness of a nonlinear optical loop mirror (NOLM) based on standard communication fiber with randomly varying birefringence for demultiplexing streams of picosecond pulses at $100 \mathrm{GHz}$. A broad switching window of about three pulse full-width at half maximum (FWHM) can be obtained. The device performance is defined by the pulse duration, the dispersion of the fiber, and the fiber length. We show that imperfect averaging of the randomly varying birefringence causes amplitude fluctuations on the NOLM transmission curve. We also show that the Raman self-frequency shift does not affect the NOLM switching characteristics at picosecond pulse durations.
\end{abstract}

\section{INTRODUCTION}

A LL-OPTICAL demultiplexers are key devices in proposed high-data-rate, all-optical, time-divisionmultiplexed transmission systems with bit rates up to 100 $\mathrm{Gb} / \mathrm{s}$. The nonlinear optical loop mirror (NOLM) is a promising demultiplexer [1]-[5]. The NOLM uses crossphase-modulation between co-propagating control and signal pulses to switch the signal pulse from one output arm to the other. Recently, several NOLM experiments were reported that use orthogonally polarized signal and control pulse streams in a standard telecommunication fiber with randomly varying birefringence [6], [7]. The main advantage of this type of NOLM is that it is simple to design and has lower loss than a NOLM based on cross-spliced polarization-maintaining fibers [5]. In this paper we present a theoretical model of a NOLM based on a fiber with randomly varying birefringence. Through numerical simulations we find the parameter regimes in which the NOLM has a broad switching window of about three times the signal pulse full-width at half maximum (FWHM). We also show that imperfect averaging of the randomly varying birefringence causes small amplitude oscillations on the switching curve that will decrease the quality of the NOLM performance. Finally, we show that the Raman effect has no visible impact for the picosecond pulse durations that we are considering.

Manuscript received September 3, 1996. This work was done at the University of Maryland and was supported by DOE, NSF, and ARPA through AFOSR.

D. Wang, E. A. Golovchenko, A. N. Pilipetskii, and C. R. Menyuk are with the Department of Computer Science and Electrical Engineering, University of Maryland, Baltimore, MD 21250 USA.

M. F. Arend is with the Naval Research Laboratory, Code 5671, Washington, DC 20237 USA.

Publisher Item Identifier S 0733-8724(97)01983-X.

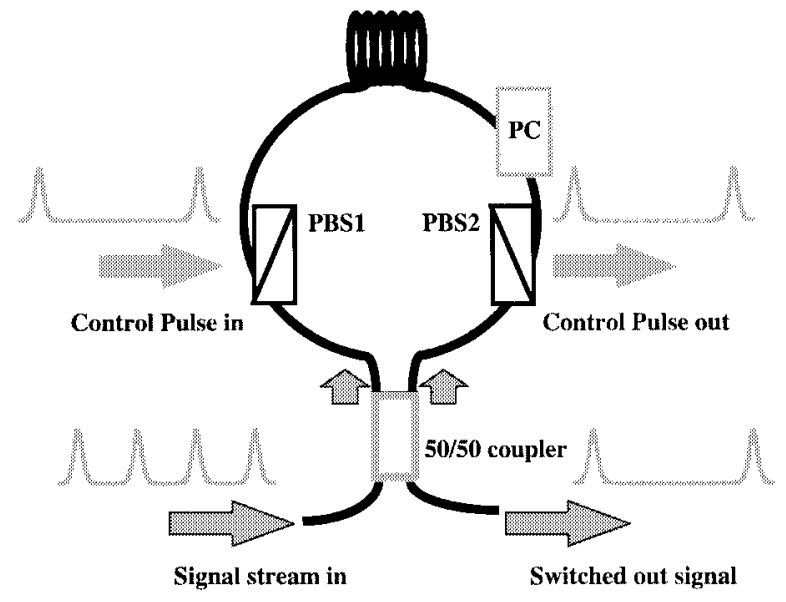

Fig. 1. Schematic illustration of a NOLM based on standard communication fiber with randomly varying birefringence; PBS1 and PBS2 indicate the polarization beam splitter, and PC indicates the polarization controller.

\section{NOLM MODEL}

We show a schematic illustration of the NOLM in Fig. 1. The signal pulse stream to be demultiplexed enters the signal-in port of the device and is divided into two counterpropagating pulse streams with equal power by a $50 / 50$ coupler. The control pulse is coupled into the loop through the polarization beam splitter PBS1 and propagates along the clockwise direction. The signal and control pulses have orthogonal states of polarization. In the absence of the control pulse, the device acts as a mirror and the signal is returned to the input. Due to the cross-phase-modulation between the control and signal pulses, the clockwise propagating signal pulse acquires a different phase shift from the counterclockwise propagating pulse. Thus, when the two signal pulses interfere at the coupler the signal pulse can be switched out from the signal-out port of the NOLM.

We are modeling a NOLM based on standard communication fiber. As long as the dispersive and nonlinear scale lengths are long compared to the fiber correlation and fiber beat lengths, a light pulse that is injected in a single polarization state will remain almost completely in a single polarization state as a function of time, although this state changes continually as the pulse evolves along the fiber. Thus, it is possible by appropriately setting the polarization controller (PC) shown in Fig. 1 to restore the control pulse to a linearly polarized state, after which it may be removed by use of the polarizing beam splitter, PBS2, leaving only the signal pulse. 
The propagation of the orthogonally polarized signal and control pulses in a fiber with randomly varying birefringence is governed by the coupled nonlinear Schrödinger equation [8]

$$
\begin{aligned}
& i\left(\frac{\partial u}{\partial z}+\delta \frac{\partial u}{\partial t}\right)+\frac{1}{2} \frac{\partial^{2} u}{\partial t^{2}}+\left(|u|^{2}+\frac{2}{3}|v|^{2}\right) u \\
& \quad+\frac{1}{3} v^{2} u^{*} \exp (-i \beta z / 2)=0, \\
& i\left(\frac{\partial v}{\partial z}-\delta \frac{\partial v}{\partial t}\right)+\frac{1}{2} \frac{\partial^{2} v}{\partial t^{2}}+\left(|v|^{2}+\frac{2}{3}|u|^{2}\right) v \\
& \quad+\frac{1}{3} u^{2} v^{*} \exp (i \beta z / 2)=0
\end{aligned}
$$

where $u$ and $v$ are the normalized amplitudes of the field along the local polarization eigenaxes of the fiber. The distance $z$ and the time $t$ are given in soliton units, in which case the length is normalized to the dispersion length $z_{d}=$ $\left[t_{0}(\mathrm{FWHM}) / 1.76\right]^{2}\left(2 \pi c / D \lambda_{0}^{2}\right)$ where $D$ is the fiber dispersion in ps $/ \mathrm{nm}-\mathrm{km}, \lambda_{0}$ is the wavelength, $c$ is the speed of light, and $t$ is normalized by pulse width $t_{0} / 1.76$. The quantity $2 \delta$ is the inverse group velocity difference which equals $2 z_{d} \Delta n / t_{0} c$ and $\beta$ is the wavenumber difference between the two polarization eigenstates at the same central frequency.

Since the orientation of the axes of birefringence in the fiber change randomly along the fiber length, we assume in our simulation that the axes periodically undergo a sudden rotation $\theta$ after every interval $\Delta z$ chosen such that

$$
\theta=\left(\frac{6 \Delta z}{z_{\text {fiber }}}\right)^{1 / 2} \operatorname{ran}(-1,1)
$$

where $\operatorname{ran}(-1,1)$ is a random number in the interval $(-1,1)$, and $z_{\text {fiber }}$ is the fiber decorrelation length. After rotation, the new field $\left(u^{\prime}, v^{\prime}\right)$ is given by

$$
\left(\begin{array}{l}
u^{\prime} \\
v^{\prime}
\end{array}\right)=\left(\begin{array}{cc}
\cos \theta & \sin \theta \\
-\sin \theta & \cos \theta
\end{array}\right)\left(\begin{array}{l}
u \\
v
\end{array}\right)
$$

in terms of the old field $(u, v)^{t}$. In our simulations, we chose $\Delta z=0.15 \mathrm{~m}$ and $z_{\text {fiber }}=76 \mathrm{~m}$. The fiber correlation length corresponds physically to the length scale over which the pulse loses memory of its initial polarization state. For the $1.6 \mathrm{ps}$ pulse durations that we will be using, the soliton period is 421 $\mathrm{m}$ and the orientation will rotate many times in one period.

To simulate the polarization controller, we first simulated the propagation of a control pulse alone which is initially in a linear state of polarization. During its propagation through a fiber with randomly varying birefringence, the control pulse becomes elliptically polarized, and its state can be expressed by the complex vector $\left(u_{c}, v_{c}\right)$. The polarization controller restores a linear state of polarization from $\left(u_{c}, v_{c}\right)$, and the pulse can then be removed from the NOLM by a polarizing beam splitter. We now define a unit vector $\left(u_{p}, v_{p}\right)=\left(v_{c}^{*},-u_{c}^{*}\right) / \sqrt{I}$ where $I=\left|u_{c}\right|^{2}+\left|v_{c}\right|^{2}$, which is orthogonal to $\left(u_{c}, v_{c}\right)$. We may express the combined action of the polarization controller and the polarizing beam splitter by first noting that in the absence of a signal pulse

$$
\left(u_{c}, v_{c}\right)\left(\begin{array}{l}
u_{p} \\
v_{p}
\end{array}\right)=0
$$

When the control and signal pulses co-propagate along the fiber they will mix together due to the randomly varying birefringence. The resultant field at the input of the polarization controller can be expressed as $(u, v)$ where the polarization state is not expected to be constant as a function of time. The output signal pulse, which will be linearly polarized, can then be expressed as

$$
u_{1}=(u, v)\left(\begin{array}{l}
u_{p} \\
v_{p}
\end{array}\right) .
$$

The complex amplitude $u_{2}$ of the counter-propagating signal pulse at the end of the loop can be found analogously. The interference at the coupler is given by

$$
\begin{aligned}
u_{\text {trans }} & =\alpha^{\frac{1}{2}} u_{1}+i(1-\alpha)^{\frac{1}{2}} u_{2} \\
u_{\text {reff }} & =-i(1-\alpha)^{\frac{1}{2}} u_{1}+\alpha^{\frac{1}{2}} u_{2}
\end{aligned}
$$

where $u_{1}$ is the co-propagating pulse at the end of the loop, $u_{2}$ is the counter propagating pulse at the end of the loop, $u_{\text {trans }}$ is the radiation transmitted through the signal-out port of NOLM, and $u_{\text {refl }}$ is the reflected radiation. The coupling coefficient $\alpha$ here is 0.5 .

\section{Results AND DisCUSSIONS}

We simulate a NOLM that is $1 \mathrm{~km}$ in length with the dispersion parameter $D=2.6 \mathrm{ps} / \mathrm{nm}-\mathrm{km}$ and birefringence of $\Delta n=2 \times 10^{-7}$ which is similar to a NOLM experiment described in [6]. The counter-clockwise and clockwise propagating signal pulses as well as the control pulse were assumed to be solitons with the duration $t_{0}(\mathrm{FWHM})=1.6 \mathrm{ps}$ and were normalized with respect to the power of either the clockwise or counterclockwise signal pulses which is half the total injected signal power.

One of the main requirements for successful NOLM performance is insensitivity to the signal pulse timing jitter. As a signal pulse can arrive either earlier or later than the control pulse, the NOLM switching window, i.e., the duration of the transmitted signal power curve as a function of the delay between the signal and control pulses, should be broader than the duration of the signal pulse. For our parameters, the duration of the switching window was 2.5 FWHM of the signal pulse, and the output signal power normalized to the input signal power was over 0.8 as shown in Fig. 2. The input and the output signal pulse shapes are shown in Fig. 3. We find that the distortion is small and that the signal nearly retains its initial hyperbolic-secant form. The asymmetry of the switching curve in Fig. 2 is due to polarization mode dispersion for the particular realization of randomly varying birefringence that we studied. A different realization leads to a slightly different curve. Our simulation results are in good agreement with the experiment reported in [6].

The small dip on the switching curve in Fig. 2 appears because the value of the accumulated phase change difference between oppositely propagating signal pulses is larger than $\pi$ at zero delay. If we increase the length of the fiber loop the width of the switching window of NOLM grows logarithmically with distance as is shown in Fig. 4; however, the increase of the switching window width is accompanied by the development 


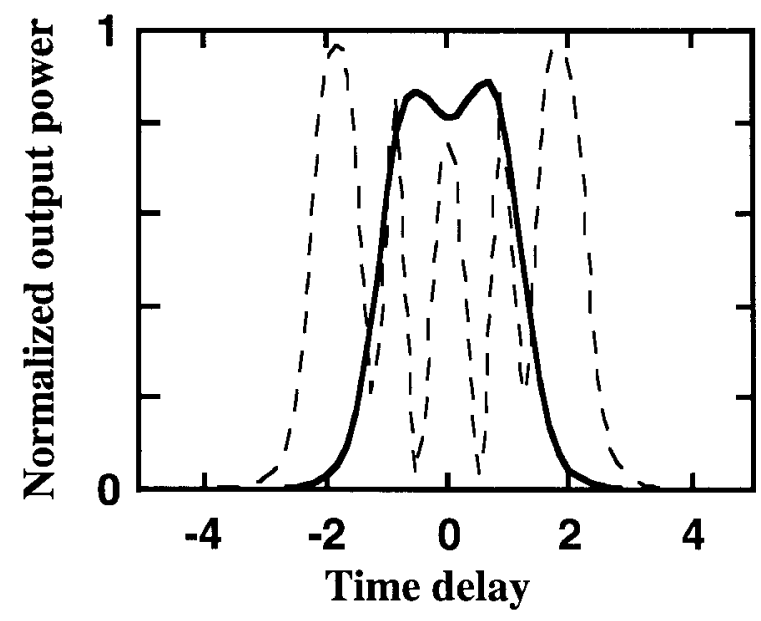

Fig. 2. The NOLM switching window curve for $t_{0}=1.6 \mathrm{ps}, \lambda=1.5 \mu \mathrm{m}$, and $D=2.6 \mathrm{ps} / \mathrm{nm}-\mathrm{km}$. The solid line corresponds to a $1 \mathrm{~km}$ length NOLM, and the dashed line corresponds to a $4 \mathrm{~km}$ length NOLM. The time delay is normalized to the input pulse duration.

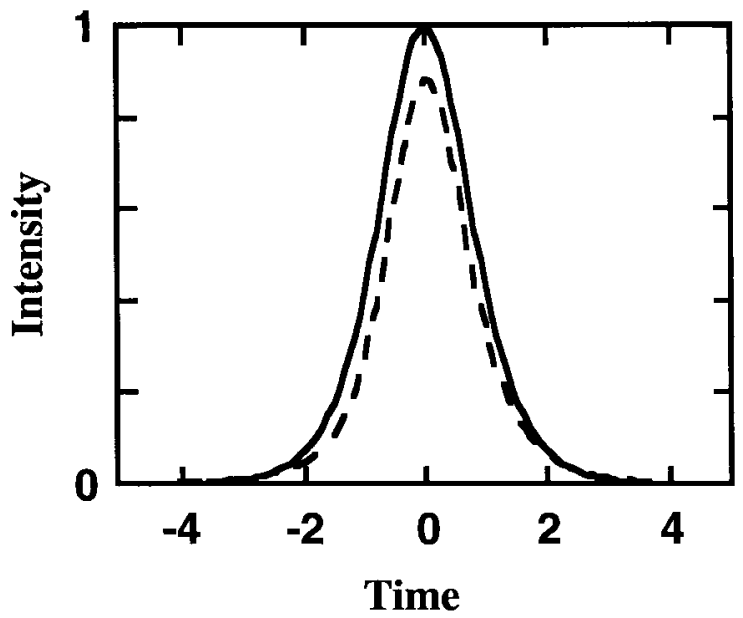

Fig. 3. The shape of the signal pulse at the input of the NOLM (solid line) and at the output of the NOLM (dashed line).

of large oscillations on the switching curve as the phase difference between oppositely propagating signal pulses continues to increase far beyond $\pi$. It is obvious that for the needs of signal demultiplexing the large oscillations on the switching window must be avoided. The phase difference between the counterpropagating signal pulses should thus be smaller than $1.5 \pi$. From our simulations we found that the broadest switching window of about three pulse FWHM can be obtained when the length of the fiber is close to five pulse dispersion lengths which corresponds to a $1.375 \mathrm{~km}$ fiber in our case.

It should be noted that when the length of the fiber is sufficiently long the pulse evolution can be averaged over all the polarization states on Poincaré sphere [8], [10]. We found that for fibers longer than $3 \mathrm{~km}$, NOLM performance can be exactly described by the averaged Manakov equations [6], [7].

When the signal and control pulses have the same power, the performance of NOLM is determined by the ratio between the fiber length and the pulse dispersion length $z_{d}$. We found that the optimum fiber length to obtain a broad NOLM switching window like that shown by the solid line in Fig. 2 should be

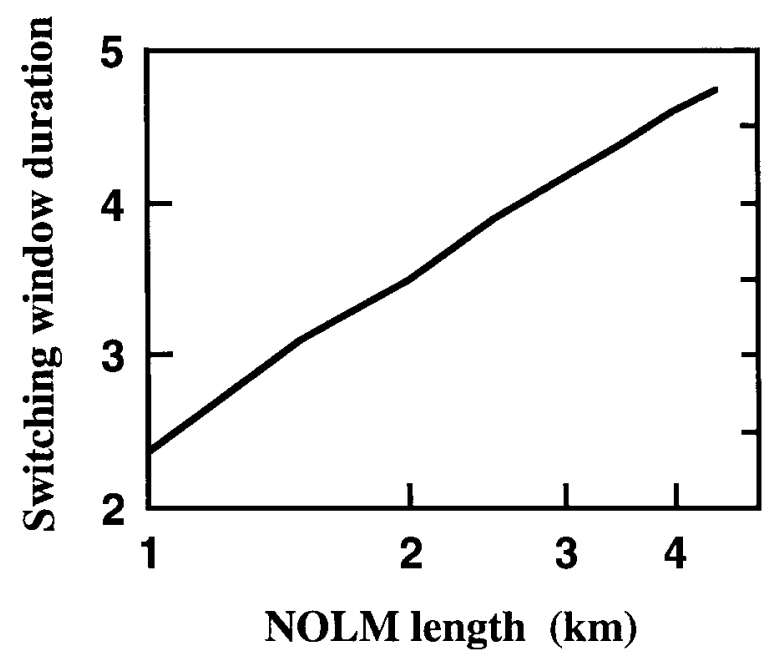

Fig. 4. The duration of the switching window normalized to the signal pulse duration versus fiber length in $\mathrm{km}$.

around four to five pulse dispersion lengths. For example, for 0.5 and 1 ps solitons, similar performance will be obtained at fiber lengths $0.2 \mathrm{~km}$ and $0.4 \mathrm{~km}$ respectively. The change of the fiber dispersion $D$ will also require a change in the fiber length.

Since in a communication line the phase of signal pulses will change due to a variety of random factors, the signal and control pulses will have a phase mismatch. To account for this phase mismatch in our simulations, we introduced a random phase difference between the control and signal pulses. Though NOLM performance is not affected significantly, and the switching curves have the same characteristics as when there is zero phase mismatch, amplitude fluctuation appear, as shown in Fig. 5. Similar fluctuations have been experimently observed [13]. These amplitude oscillations are due to imperfect averaging of the nonlinear terms due to the randomly varying birefringence, an effect that is called nonlinear polarization mode dispersion [12]. When the pulse evolution is averaged over all the polarization states the amplitude oscillations disappear. Fig. 5 shows that the switching curve obtained by using the Manakov equation instead of (1) lies at the mean level of oscillations.

Besides the phase mismatch, the control and signal pulses can have an amplitude mismatch. For these calculations we used a $1 \mathrm{~km}$ fiber, and the pulse FWHM was $1.6 \mathrm{ps}$. When the signal pulse power is larger than the control pulse power, the NOLM performance does not degrade, but when the control pulse has more power than the signal pulse, the NOLM output power decreases significantly. Fig. 6 shows the switching curves for different power ratios between the control and signal pulses. For a signal pulse power that is 1.3 times as large as the control pulse power, the width of the switching window is almost the same as when there is no power difference and the normalized output power is larger than 0.8. The dip at the center of the window is deeper than the dip that appears when the control-to-signal pulse power ratio is 1 to 1 . If the ratio between the signal and control pulse powers is 1 to 1.3 , the normalized output power is smaller than 0.5 , and we can conclude that the device performance is unacceptable. 


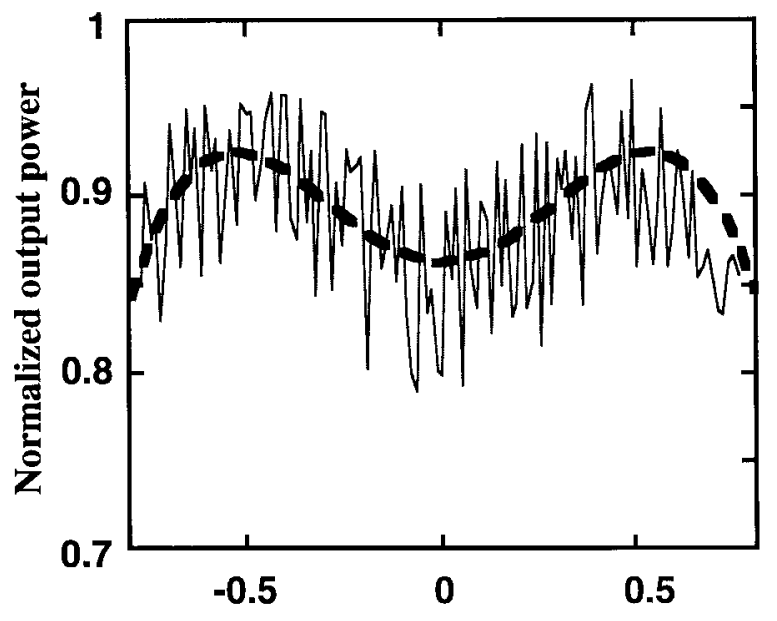

Time delay

Fig. 5. The NOLM switching window with random phase mismatch introduced between the control and signal pulses at the input of the NOLM (solid line). The dashed line shows the same switching curve but calculated using the Manakov equation.

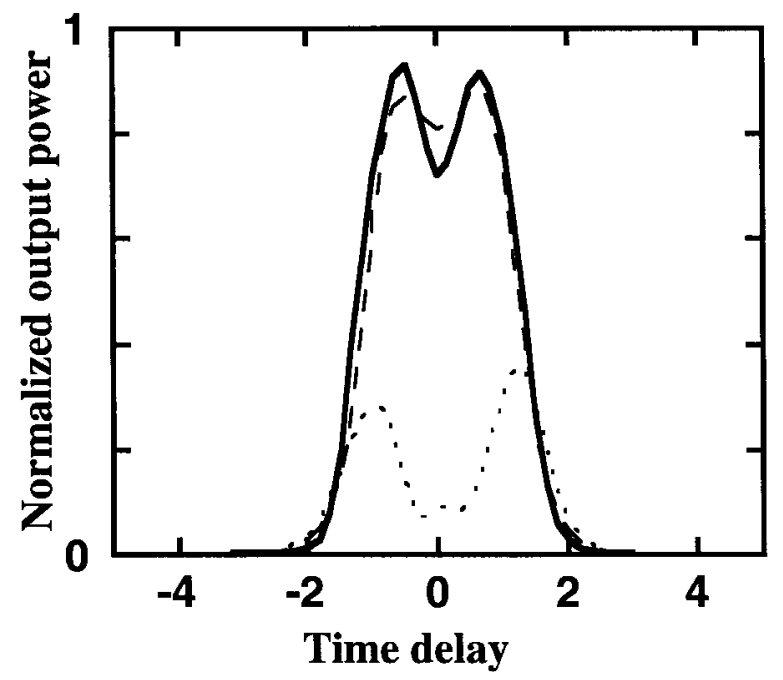

Fig. 6. Switching window for different pulse powers. The ratio between the signal pulse power $|u|^{2}$ to control pulse power $|v|^{2}$ is $1: 1$ (solid line); $|u|^{2}:|v|^{2}=1: 1.3$ (dotted line); $|u|^{2}:|v|^{2}=1.3: 1$ (dashed line).

For the results that we have discussed up to this point, polarization mode dispersion is almost negligible, but that will not be the case if fibers with a larger average birefringence strength are used. Fig. 7 compares NOLM performance for the values of birefringence $\Delta n=10^{-7}$ and $\Delta n=10^{-5}$. For $\Delta n=10^{-5}$ the switching window becomes highly asymmetric, and the normalized output power is significantly decreased.

The last issue that we will discuss here is the effect of the pulse Raman self-frequency shift on the NOLM performance. Pulse propagation in the fiber for these calculations was modeled by using the modified coupled nonlinear Shrödinger equations which included the response functions for both the parallel and the orthogonal Raman contributions to the nonlinear susceptibility [11]. We found that even when the pulse duration is decreased to $0.1 \mathrm{ps}$ the characteristics of

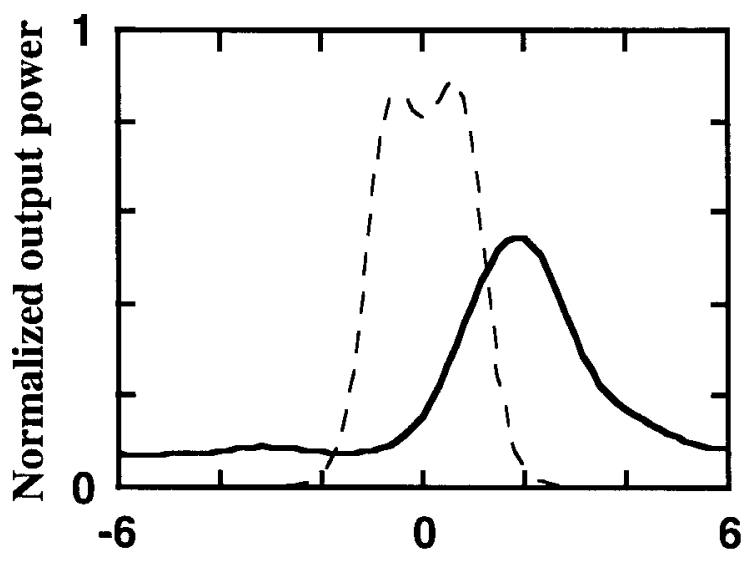

\section{Time delay}

Fig. 7. The NOLM switching window for different values of the birefringence strength; $\Delta n=1 \times 10^{-5}$ (solid line), $\Delta n=2 \times 10^{-7}$ (dashed line).

the NOLM performance do not change significantly, and the shape and the width of the switching curve in units of the pulse FWHM do not differ much from those shown in Fig. 2 .

\section{CONCLUSION}

The NOLM based on a randomly varying birefringence fiber is a promising device for high-speed all-optical switching and demultiplexing because of its simplicity and effectiveness. Our calculations showed that operation of this type of NOLM is tolerant to pulse timing and amplitude jitter. We found that when the fiber loop length is around three to four signal pulse dispersion lengths a broad and smooth switching window of about two to three pulse widths can be obtained. The device has a stable performance when the signal pulse power is larger than the control pulse power, but is sensitive to the increase of the control pulse power versus signal pulse power. The output signal power is sensitive to the initial phase mismatch between the signal and control pulses. This sensitivity may degrade the device performance. Our calculations showed that this type of NOLM can work well in the subpicosecond pulse duration range and is not affected by the Raman self-frequency shift.

\section{ACKNOWLEDGMENT}

The authors would like to thank M. L. Dennis, I. N. Duling, and P. K. A. Wai for helpful discussions.

\section{REFERENCES}

[1] T. Morioka, M. Saruwatari, and A. Takada, "Ultra-fast optical multi/ demultiplexer using optical Kerr effect in polarization-maintaining fibers,' Electron. Lett., vol. 23, pp. 453-454, 1987.

[2] P. A. Andrekson, N. A. Olsson, J. R. Simpson, T. Tanbun-Ek, R. A. Logan, and M. Hanner, "16 Gbit/s all-optical demultiplexing using four-wave mixing," Electron. Lett., vol. 27, pp. 922-924, 1991.

[3] N. A. Whittaker Jr., H. Avramopoulos, P. M. W. French, M. C. Gabriel, R. E. LaMar.e, D. J. Di Giovanni, and H. M. Presby, "All-optical arbitrary demultiplexing at $2.5 \mathrm{Gbits} / \mathrm{s}$ with tolerance to timing jitter," Opt. Lett., vol. 16, pp. 1838-1840, 1991. 
[4] N. J. Doran and D. Wood, "Nonlinear optical loop mirror," Opt. Lett., vol. 13 , pp. 56-58, 1988.

[5] G. R. Williams, M. Vaziri, K. H. Ahn, B. C. Barnett, M. N. Islam, K. O. Hill, and B. Malo, "Soliton logic gate using low-birefringence fiber in a nonlinear loop mirror," Opt. Lett., vol. 20, pp. 1671-1673, 1995.

[6] M. L. Dennis, M. F. Arend, and I. N. Duling III, "Soliton loop mirror demultiplexer using orthogonally polarized signal and control pulse," IEEE Photon. Technol. Lett., vol. 8, pp. 906-908, 1996.

[7] D. L. Butler, P. S. Cao, M. W. Chbat, J. Goldhar, F. S. Choa, and G. L. Burdge, "Operation of a nonlinear optical loop mirror with orthogonally polarized waves in nonpolarization-preserving, singlemode fiber," IEEE Photon. Technol. Lett., vol. 8, pp. 779-781, 1996.

[8] C. R. Menyuk, "Stability of solitons in birefringent optical fibers. II. Arbitrary amplitudes," J. Opt. Soc. Amer. B, vol. 5, pp. 392-401, 1988.

[9] M. J. Ablowitz and H. Segur, Solitons and the Inverse Scattering Transform. SIAM, Philadelphia, PA: 1981, p. 250.

[10] S. G. Evangelides, L. F. Mollenauer, J. P. Gordon, and N. S. Bergano, "Polarization multiplexing with solitons," J. Lightwave Technol., vol. 10, pp. 28-35, 1992.

[11] C. R. Menyuk, J. P. Gordon, and M. N. Islam, "Raman effect in birefringent optical fibers," Opt. Lett., vol. 16, pp. 566-568, 1991; see also, E. A. Golovchenko and A. N. Pilipetskii, "Theoretical analysis of spectrum generation by modulational instability and Raman amplification in highbirefringent fibers," Sov. Lightwave Commun., vol. 1, pp. 271-283, 1991.

[12] P. K. A. Wai and C. R. Menyuk, "Polarization mode dispersion, decorrelation and diffusion in optical fibers with randomly varying birefringence," J. Lightwave Technol., vol. 14, pp. 148-157, 1996.

[13] M. F. Arend, M. L. Dennis, I. N. Duling III, E. A. Golovchenko, A. N. Pilipetskii, and C. R. Menyuk, "Simulations of a nonlinear optical loop mirror demultiplexer using random birefringence fiber," in $O F C$ ' 96 Tech. Dig., pp. 226-227.

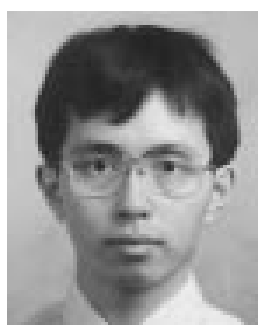

Ding Wang was born on June 8, 1972. He received the B.S. degree in technology of opto-electronics from the University of Electronic Science and Technology of China (UESTC) in 1993. He is currently a graduate student in the Department of Computer Science and Electrical Engineering in the University of Maryland, Baltimore County.

He studied and worked in the Institute of Applied Physics at UESTC, which included fast-spectrum discerning and air optical communication. He is a Research Assistant to Dr. C. R. Menyuk's group in the Computational Photonics Laboratory. His current research interests are in theoretical and computational studies of fiber optic communications and nonlinear effect in optical fiber.

Mr. Wang is a student member of OSA.

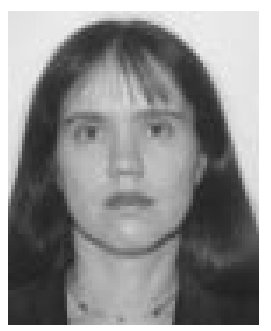

Ekaterina A. Golovchenko was born in 1962. She received the B.S. degree in 1982 and the M.S. degree from Moscow State University, Moscow, Russia, in 1985. She received Ph.D. from the General Physics Institute, Russian Academy of Sciences in 1991.

In 1985 she joined the Fiber Optics Department of the General Physics Institute, Academy of Sciences of the U.S.S.R, Moscow, as a Research Scientist. She is now the Assistant Research Professor of University of Maryland, Baltimore County now. Her research interests include Raman stimulated scatterings, Raman selfscattering, ultrashort pulse generation, solitons, theoretical and computational research of optical fiber communication. She has authored or coauthored more than 60 papers and conference presentations.

Dr. Golovchenko is a member of OSA.

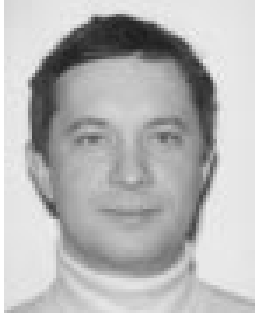

Alexei N. Pilipetskii was born in 1962. He received the B.S. degree in 1982 and M.S. degree from Moscow State University, Moscow, Russia, in 1985. He received Ph.D. degree from the General Physics Institute, Russian Academy of Science of the U.S.S.R, Moscow, as a Research Scientist.

$\mathrm{He}$ is currently a Research Professor of University of Maryland, Baltimore County. His research interests include Brillouin and Raman stimulated scattering, ultrashort pulse generation, solitons, and modulational instability in optical fibers. He has authored or coauthored more than 80 papers and conference presentations.

Dr. Pilipetskii is a member of OSA.

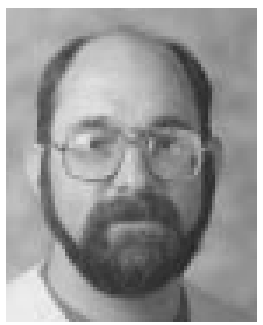

Curtis R. Menyuk (SM'88) was born March 26, 1954. He received the B.S. and M.S. degrees from the Massachusetts Institute of Technology (M.I.T.), Cambridge, in 1976 and the Ph.D. degree from the University of California at Los Angeles (UCLA), in 1981.

He has worked as a Research Associate at the University of Maryland, College Park, and at Science Applications International Corporation in McLean, VA. In 1986, he became an Associate Professor in the Department of Electrical Engineering at the University of Maryland, Baltimore County, and he was the founding member of this department. In 1993, he was promoted to Professor. For the last few years, his primary research area has been theoretical and computational studies of fiber optic communications. He has authored or coauthored more than 100 publications, one book, and two patents. He has written computer codes to model the nonlinear propagation of light in optical fibers which have been used by industry, government, and university research groups throughout the United States.

Dr. Menyuk is a member of APS and SIAM and a fellow of OSA.

Mark F. Arend, photograph and biography not available at the time of publication. 